\title{
Coulisses
}

Revue de théâtre

13 | Hiver 1996

Varia

\section{Une pièce de Jules Verne : Voyage à travers \\ l'impossible (1882)}

\section{Florent Montaclair}

\section{(2) OpenEdition}

1 Journals

Édition électronique

URL : http://journals.openedition.org/coulisses/3978

DOI : $10.4000 /$ coulisses.3978

ISSN : 2546-9460

Éditeur

Presses universitaires de Franche-Comté

\section{Édition imprimée}

Date de publication : 1 janvier 1996

Pagination : 21-28

ISSN : 1150-594X

\section{Référence électronique}

Florent Montaclair, «Une pièce de Jules Verne : Voyage à travers l'impossible (1882) », Coulisses [En ligne], 13 | Hiver 1996, mis en ligne le 15 mars 2019, consulté le 01 octobre 2020. URL : http:// journals.openedition.org/coulisses/3978 ; DOI : https://doi.org/10.4000/coulisses.3978

Ce document a été généré automatiquement le 1 octobre 2020.

Coulisses 


\title{
Une pièce de Jules Verne : Voyage à travers l'impossible (1882)
}

\author{
Florent Montaclair
}

1 Le thème de la science est dans les romans de Jules Verne majeur. Son expression passe par le dialogue des personnages, par le rappel de découvertes passées, par l'exposition de théories savantes, par l'intervention, dans l'histoire, de scientifiques réels. Dans les romans, la science est « raisonneuse », elle s'explique et se disserte. Dans le théâtre de Verne au contraire, elle est visuelle et spectaculaire.

Lorsqu'en 1882, Jules Verne (1828-1905) écrit une pièce de théâtre, une des raisons qui motive ce retour à la scène après dix années de production romanesque, est de monter une œuvre où la science prend une couleur de légèreté. Jules Verne veut se dégager des conventions éditoriales que faisait peser sur lui Pierre-Jules Hetzel, son éditeur. La science au théâtre se manifeste par les décors, les actions et les paroles des personnages, elle joue essentiellement sur l'imaginaire et se détache du rôle purement pédagogique et moral qu'elle avait dans le roman. Voyage à travers l'impossible est l'adaptation au théâtre de trois textes verniens mais le traitement de la donnée scientifique y est résolument différent.

\section{Les premières pièces : la science absente}

2 L'utilisation de la science dans Voyage à travers l'impossible se comprend dans l'optique des romans verniens. En effet, les premières pièces de Jules Verne, écrites avant sa rencontre avec Hetzel, n'offrent aucune perspective scientifique.

La production littéraire de Jules Verne commence au théâtre. Sa première pièce est une comédie en un acte et en vers : Les Pailles rompues. Joué douze fois au Théâtre lyrique, ce marivaudage permet à Verne de devenir secrétaire de Souvestre et d'entrer de plainpied dans le monde du spectacle. Il fait représenter quelques pièces... il participe à la création de comédies et d'opéras, et côtoie auteurs, acteurs, machinistes, compositeurs et musiciens.

Le jeune Jules Verne commence sa carrière d'écrivain au théâtre, au milieu des jeux 
d'esprit, des musiques et des ballets, des acteurs et des machinistes, entre la comédie et le drame, entre la fantaisie et la morale, dans un domaine en pleine évolution qui mélange les genres et les esthétiques. Le théâtre est fait pour distraire... La science y est absente.

Cependant, après un début d'une dizaine d'années au théâtre (1850-1861), après quelques nouvelles plus ou moins "hoffmanniennes", Jules Verne se lance entièrement dans l'écriture de romans-feuilletons, avec un but purement géographique et éducatif. Verne tentera alors deux retours au théâtre entre 1874 et 1887.

Nous ignorerons ici la première forme d'écriture théâtrale à laquelle revient Jules Verne, qui est celle de l'adaptation de ses romans. En 1874, Le Tour du monde en quatrevingts jours, "pièce en cinq actes et un prologue ", écrite avec Adolphe d'Ennery est joué au Théâtre de la Porte Saint-Martin; en 1881 Les Enfants du capitaine Grant (cinq actes), texte de Jules Verne et Adolphe d'Ennery, est joué au même Théâtre de la Porte Saint-Martin; il y a ensuite Michel Strogoff (cinq actes) en cette année 1881 avec le même d'Ennery au théâtre du Châtelet; en 1883 Kéraban le Têtu (cinq actes) est monté au Théâtre de la Gaieté Lyrique et en 1887, Mathias Sandorf (quatre actes) écrit avec Busnach et Maurens, est présenté au Théâtre de l'Ambigu-Comique. Le propre des Voyages Extraordinaires, appellation générique de tous les romans de Verne, était de dépayser le lecteur et de lui montrer le vaste monde... sur scène le but reste le même et les décors sont pour cela fastueux. Ecoutons François Raymond sur les mises en scène des romans verniens :

Ces Voyages au théâtre, [sont] trop oubliés aujourd'hui, où les deux compères s'étaient amusés au fil des ans à montrer, sur la scène (...) un éléphant véritable, un train à vapeur, le naufrage d'un bateau (auxquels furent ajoutés de fabuleux serpents, et quelques lions, non moins véritables à la reprise); une mirifique baleine en caoutchouc, un soleil de minuit; des chevaux, des canons, un fleuve de « naphte »- enflammé, bien entendu. ${ }^{1}$.

Les spectacles sont somptueux et connaissent un véritable triomphe. Nous laissons pourtant de côté ce type de retour au théâtre: ce sont là des récits de voyage et la science n'y a pas sa place.

Nous regardons une création plus curieuse de 1882 : Voyage à travers l'impossible. Nous n'avons pas là affaire à une simple adaptation, nous avons en une seule pièce de trois actes un syncrétisme de trois grands romans verniens. En changeant l'histoire, les personnages, les décors, les situations... en faisant du neuf avec du vieux, Jules Verne et Adolphe d'Ennery montent une pièce née de Voyage au centre la terre (1863), de Vingt mille lieues sous les mers (1870), de De la Terre à la Lune (1865), reprenant le héros d'Une fantaisie du Docteur Ox (1872), donnant naissance au fils du fameux capitaine Hatteras (Les Aventures du capitaine Hatteras, 1863)... Voyage à travers l'impossible est une pièce syncrétique, synthétique ${ }^{2}$. L'utilisation qui est faite de la donnée scientifique dans cette pièce, est à mettre en rapport avec la forme qu'elle prenait dans les romans de Jules Verne. 


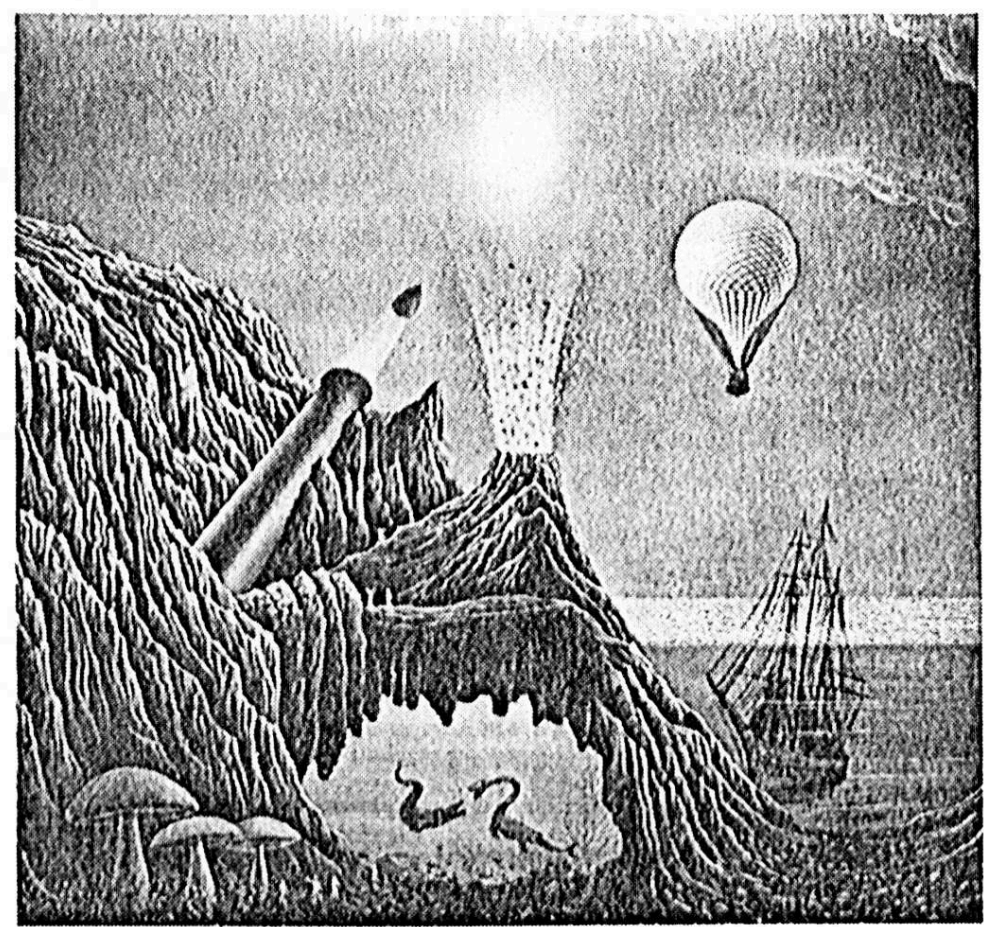

\section{La science et les volontés éditoriales}

5 Les données scientifiques présentes dans les romans de Jules Verne sont en demiteinte, à la fois onirisées et rationalisées.

$\mathrm{Au}$ milieu du XIX ${ }^{\text {ème }}$ siècle, les découvertes scientifiques se multiplient: le public devient friand de récits, fictionnels ou non, qui rendent compte des extraordinaires perspectives qu'ouvre la modernité. L'éditeur de Jules Verne, Pierre-Jules Hetzel rend compte dans l'introduction de son Magazine d'éducation et de récréation de cet enthousiasme pour la science :

Quand on voit le public empressé courir aux conférences qui se sont ouvertes sur mille points de France, quand on voit qu'à côté des critiques d'art et de théâtre il a fallu faire place dans nos journaux aux comptes rendus de l'Académie des sciences, il faut bien se dire que l'art pour l'art ne suffit plus à notre époque, et que l'heure est venue où la science a sa place faite en littérature. (...) Les ouvrages parus et ceux à paraître embrasseront ainsi dans leur ensemble le plan que s'est proposé l'auteur (...) son but est en effet de résumer toutes les connaissances géographiques, géologiques, physiques, astronomiques, amassées par la science moderne, et de refaire, sous la forme attrayante qui lui est propre, l'histoire de l'univers ${ }^{3}$.

Les magazines alternant nouvelles, feuilletons, articles scientifiques sont nombreux : Pitre-Chevalier fonde le Musée des Familles, lecture du soir (1850), Bry publie les Veillées littéraires illustrées (1849) et Barba le Panthéon populaire illustré (1850), Hetzel crée en 1860 la Bibliothèque d'éducation et de récréation. Trois constantes dirigent les volontés éditoriales : les collections populaires doivent éduquer la jeunesse, elles doivent être agrémentées de jolies gravures («faciles à lire» dit Barba) et elles doivent publier les " grands classiques de la littérature de tous les temps » (Bry, introduction aux Veillées 
illustrées).

Dans ces conditions, la description scientifique et le dialogue entre le héros et le savant - figure typique du roman vernien - sont là pour éduquer et renseigner. Ainsi Lidenbrock explique-t-il la formation de la terre à Axel par l'exposé d'une théorie de Davy (1778-1829) et par le rappel d'une expérience scientifique qu'il fit jadis :

- Mais il semble pourtant évident que la surface du globe a été soumise à la combustion, et il est permis de supposer que la croûte extérieure s'est refroidie d'abord, tandis que la chaleur se réfugiait au centre.

- Erreur, répondit mon oncle; la terre a été échauffée par la combustion de sa surface, pas autrement. Sa surface était composée d'une grande quantité de métaux, tels que le potassium, le sodium, qui ont la propriété de s'enflammer au seul contact de l'air et de l'eau; ces métaux prirent feu quand les vapeurs atmosphériques se précipitèrent en pluie sur le sol ; et peu à peu, lorsque les eaux pénétrèrent dans les fissures de l'écorce terrestre, elles déterminèrent de nouveaux incendies avec explosions et éruptions. De là les volcans si nombreux aux premiers jours du monde.

- Mais voilà une ingénieuse hypothèse ! m'écriai-je un peu malgré moi.

- Et qu'Humphry Davy me rendit sensible, ici même, par une expérience bien simple. Il composa une boule métallique faite principalement des métaux dont je viens de parler, et qui figurait parfaitement notre globe ; lorsqu'on faisait tomber une fine rosée à sa surface, celle-ci se boursouflait ${ }^{4}$.

7 Le texte développe des théories scientifiques, décrit des expériences... On apprend le fonctionnement d'appareils précis, on retient des faits historiques (découverte de planètes nouvelles, construction de ballons dirigeables, transformations techniques majeures comme l'amélioration des méthodes de fonte de l'acier...), on admire de grands précurseurs du domaine scientifique.

Cependant, par-delà le pôle éducatif et l'utilisation de la science à fin pédagogique, les romans verniens doivent abreuver l'imaginaire à des sources nouvelles, et dans leurs voyages, les personnages se trouvent bien vite confrontés à la petitesse du savoir de l'homme sur son environnement. D'où une utilisation onirisée des sciences... ou plus exactement un jeu constant sur une extrapolation contrôlée des théories scientifiques. Jules Verne n'invente jamais rien, ni le sous-marin, ni l'aviation, ni le cinéma ni quoi que ce soit dont on l'a crédité... car dans cet ordre d'idée rappelons qu'il invente des îles automobiles qui dérivent à volonté sur l'eau, des trains d'obus qui permettent de voyager dans l'espace, des locomotives sans rails... Jules Verne n'était pas un visionnaire et l'utilisation qu'il faisait des sciences reposaient essentiellement sur leur confrontation. Dans Voyage au centre de la terre, deux théories s'opposent : l'une veut que la terre soit creuse (et Lidenbrock y croit) l'autre veut que le centre de la terre soit composé de laves. L'expérience des héros donne raison à la théorie de la terre creuse mais Axel, le jeune héros garde encore à la fin du roman certains doutes : «je ne puis admettre sa théorie du refroidissement : en dépit de ce que j'ai vu, je crois et je croirai toujours à la chaleur centrale $»^{5}$.

8 L'effet de surprise et d'étrangeté vient d'abord de la mise en défaut de la science, puis de sa réaffirmation massive. Le savoir technique est à ce point merveilleux qu'il peut se contredire et s'affirmer à la fois. La science luttant contre la science accentue l'effet extraordinaire. Le centre de la terre est lieu magique, et l'aventure le devient aussi. Le vide central du globe s'explique rationnellement mais il est un détracteur scientifique de la science.

La science a donc deux fonctions dans les romans verniens : être instructive - ce sont les dialogues des personnages et les descriptions qui traduisent cette fonction - et être 
satisfaisante pour l'imaginaire, revêtir un caractère magique ou fantastique - c'est la confrontation de théories scientifiques admises qui permet cette fonction.

$\mathrm{Au}$ théâtre, le cas est un peu différent. Les exigences de la scène ne sont pas celle du roman et le traitement de la donnée scientifique est donc plus fantaisiste.

\section{Le mélodrame : la distraction et le « visuel »}

9 Jules Verne affectionne un type de représentation théâtrale très particulier, qui mêle la comédie et le drame, la musique et le texte, les acteurs et les danseurs. Verne veut distraire le public... il supprime donc de ses textes tout dialogue compliqué, toute description censée transmettre un savoir, tout rappel d'invention passée. Le spectateur n'est pas comme le jeune héros, il ne vient pas recevoir une leçon. La première dimension de la science dans les romans de Jules Verne n'a donc pas lieu d'être: le théâtre vernien n'a pas les visées pédagogiques du feuilleton. Les grands noms de scientifiques, les expériences et les découvertes, les inventions et les débats sont absents. Le rythme de tel passage est jugé par Verne trop peu énergique, trop long et trop ennuyeux. Les dialogues de Voyage à travers l'impossible sont en effet plus toniques et jouent sur le mélange du drame et de la comédie. D'une situation cruelle à la Roméo et Juliette on passe à une franche bouffonnerie. Deux couples s'opposent dans Voyage à travers l'impossible: Eva aime Georges Hatteras, qui ne pense qu'à parcourir le monde pour découvrir les lieux extrêmes où nul n'est allé. Ox, amoureux d'Eva, décide de conduire Georges au centre de la terre, sous les mers, dans les étoiles, en espérant qu'il y laissera la vie. A ce trio tragique, dans lequel ox menace souvent de tuer Georges si Eva ne cède à ses avances, s'oppose celui, comique, de Valdemar, jeune désargenté à la recherche de la fortune, désireux d'épouser Mlle Babichok, surveillée au Danemark par le cousin Finderup. Lorsqu'il sera riche, Valdemar apprendra que son cousin a épousé la belle Babichok... lorsqu'il redeviendra pauvre, une dépêche miraculeuse lui apprendra la mort de Finderup, étranglé par une arête de poisson au cours de son repas de noce. Valdemar retrouve sa fortune, rentre au Danemark et épouse enfin l'inconstante Babichok. Eva, elle aussi, épousera son aimé, la fin est donc joyeuse... mais le premier couple est tragique, le second comique.

$\mathrm{ox}$ : Je veux une dernière fois tenter de vous fléchir et d'attendrir votre coeur...

EVA: Et votre coeur à vous s'est-il attendri? Avez-vous donc cessé de me persécuter?

ox : Ce n'est pas toi !... c'est lui !... mon rival détesté...

EVA : Mais, lui, c'est toute ma vie.

ox : Ne me dis pas cela!

EVA : Lui, c'est toute mon âme.

ox : Tais-toi. »

EVA : Lui, c'est tout mon bonheur, tout mon amour..

ox (avec force) : Assez ! assez, te dis-je!

EVA : Et c'est en l'égorgeant que vous voulez arriver jusqu'à moi !... Ah! vous m'assassinez, et vous voulez que je vous aime !... Eh! bien, sachez-le donc, tout ce que vous ressentez d'aversion pour Georges que j'adore, je le ressens pour vous... vous le haïssez... je vous hais. ${ }^{6}$

10 Couple tragique, scène de déchirement, héroïne fragile et forte résistant à la menace... les scènes de drame sont nombreuses et s'opposent aux évocations comiques du trio Valdemar, Babichok, Finderup, auquel s'ajoute Tartelet : 
TARTELET : M. Valdemar, prenez garde!

VALDEMAR: Prenez garde vous-même, et n'oubliez pas que sur cette planète, ma force est décuplée !...

TARTELET : La mienne aussi, je suppose !... Et la preuve... Vlan !... Tant pis ça y est ! (Il

lui envoie un vigoureux coup dans le derrière. Valdemar s'élève à 2 mètres du sol.)

VALDEMAR : Hein... Qu'est-ce que cela veut dire? (il retombe).

TARTELET (riant) : Ah!... ah!... ah!... Le manque d'attraction. Les pieds en dehors, Monsieur, les pieds en dehors !

VALDEMAR: Ah! Scélérat! (Il lui envoie à son tour un coup de pied pareil.) Tartelet

(s'élevant de même) : Ah !... (il retombe).

VALDEMAR : Le manque d'attraction, Monsieur, le manque d'attraction! Ah! Chut !

du monde !

La critique apprécia les personnages comiques (Valdemar et Tartelet); et les effets comiques-dramatiques qui s'enchaînent dans la double intrigue des couples opposés contentèrent vraisemblablement le public ${ }^{8}$. Le retour de Jules Verne au théâtre avec Voyage à travers l'impossible est un retour au théâtre en tant que lieu, avec son public, ses acteurs et ses décors. Verne veut la fantaisie et la distraction, non l'éducation et la vraisemblance. La science n'est donc jamais utilisée dans une dimension pédagogique. Ce que Verne jugeait bon d'analyser avec force détails techniques dans ses romans (la possibilité d'une terre creuse, l'invention d'un sous-marin, le départ vers les étoiles), n'est jamais expliqué. Les personnages pénètrent le centre de la terre, voyagent sous l'eau, dans le cosmos, dans le temps et l'espace grâce à une potion inventée par Ox :

Vois ce flacon, quiconque aura bu quelques gouttes de cette liqueur, sera emporté avec la rapidité de la foudre et dans les conditions d'une vie nouvelle jusqu'aux milieux interdits à l'homme! Plus d'intervalle de temps, plus d'intervalle des distances! On vole, prompt comme l'éclair, les jours s'écoulent en quelques secondes, les années en quelques minutes (...) j'aurai donné à ton corps la faculté de ne pas brûler où l'on brûle, de ne pas se noyer là où on se noie, de respirer là où il n'y a plus d'air. ${ }^{9}$

12 Aucune autre explication n'est fournie.

La dimension scientifique est pourtant omniprésente dans la pièce : ce n'est pas une science rationnelle, expliquée, c'est une science onirisée, futuriste. Parce que la dimension éducative du feuilleton ne dirige plus l'écriture vernienne, la pièce extrapole de toute part ce que le savoir scientifique laisse supposer : les découvertes faites par les aventuriers sont révolutionnaires.

La dimension magique de la science est excessivement présente dans la pièce. Cependant, elle n'est pas le produit d'une confrontation des savoirs technologiques, elle est une donnée purement visuelle. La science s'exprime par le décor, les costumes et le jeu des personnages. Dans le dialogue cité plus haut entre Tartelet et Valdemar, l'existence de l'apesanteur est montrée au cours d'une dispute entre les personnages... une scène comique et un jeu de poulies et de cordes permettent de faire comprendre plaisamment au public ce qu'est l'attraction terrestre.

La science s'expose aussi par les scènes de ballet et de musique. Voyage à travers l'impossible est un mélodrame ${ }^{10}$, le texte est de Jules Verne et d'Adolphe d'Ennery, les nombreux ballets et les intermèdes musicaux sont signés Oscar de Lagoanère. Les trois actes de la pièce présentent vingt tableaux différents (dont seuls dix-sept seront retenus dans la version finale) et les scènes charnières - qui passent d'un acte à l'autre ou d'un tableau à l'autre - sont introduites ou finies par des pièces musicales à un ou plusieurs instruments, par des ballets et des jeux de scène plus ou moins chorégraphiques. A l'acte I, $4^{\circ}$ tableau, scène I, Tartelet, le professeur de danse, au cœur 
de notre planète, découvre la majestueuse sonorité des cavernes souterraines : "il prend son violon et joue une gavotte avec une puissance de son surprenante " nous disent les didascalies. Et un critique de l'époque, spectateur de la pièce, déclare avoir entendu « un Stradivarius manié par un Paganini »" ${ }^{11}$. Le spectateur n'a pas, comme le lecteur, besoin de savantes métaphores ou de longs paragraphes pour comprendre les conséquences des théories scientifiques sollicitées par les personnages. Les ballets qui accompagnent le texte accentuent son côté magique et montrent l'extraordinaire des découvertes faites par les personnages. Ce que Verne ne produisait qu'à force de descriptions dans le Voyage au centre de la terre ${ }^{12}$, naît ici soudainement, du mélange si théâtral du son, du geste et du texte.

La science est montrée par la musique, la danse et les décors. Robert Pourvoyeur cite une lettre de Hetzel-fils à Hetzel-père dans laquelle la désapprobation face à la pièce de 1882 est totale : «C'est une insanité et quand même le livre ou la pièce serait un chefd'œuvre, le titre serait [à lui] seul un coup porté à ce produits " ${ }^{13}$. Les deux Hetzel désapprouvent le projet de Voyage à travers l'impossible parce que cette pièce présente un certain nombre d'éléments relevant de la science mais sans aucune attache avec une réalité démontrable. La pièce suggère ce que la science poussée à ses dernières limites laisse penser. Voyage à travers l'impossible est une des rares œuvres verniennes à pouvoir porter le nom d'anticipation. Tout ce qui était prudemment avancé par les personnages de romans devient visuel, admis, acceptable. Nemo faisait visiter l'Atlantide en ruine, submergée par l'océan, à Arronax, Ox la présente vivante et resplendissante à Georges Hattéras :

La ville de Makhimos, capitale de l'Atlantide, quatre ou cinq mille ans avant l'ère chrétienne. Architecture où se mélange le Mauresque, l'Arabe et le style des hypogées mexicains.

Ce n'est pas une Atlantide de musée et de savants que le spectateur voit, c'est un pays vivant, avec ses habitants et ses cités.

Dans De la Terre à la Lune, les explorateurs du satellite terrestre déclaraient que la lune n'était pas habitée... ils ne voyaient pas comme $\mathrm{Ox}$ et ses compagnons des « extraterrestres » de la planète Altor... Dans la pièce, les didascalies nous disent :

Un site sur la planète Altor. Au lointain la silhouette d'une ville, qui paraît bâtie en or et en argent. A droite, au premier plan, la façade d'une habitation dont les murs sont incrustés de pierres précieuses

et plus loin

Immense place bordée de palais d'une architecture spéciale. Les murs sont bâtis en pierres précieuses, en marbres de la plus grande beauté. L'or et l'argent apparaissent partout. Lumière éclatante qui a toute l'intensité de la lumière électrique.

16 Là encore, l'habitabilité des planètes lointaines, qui était suggérée dans le roman par les références aux savants de l'époque ${ }^{14}$, est une donnée immédiate. Point de débats, de dissertations et de raisonnements : la scène montre ce que la science de l'époque laisse supposer mais sans aucune démonstration ni référence.

Dans Voyage au centre de la terre, Jules Verne avait mis en scène des dinosaures - mais on savait qu'ils avaient existé grâce à Cuvier - et des hommes préhistoriques - mais sous forme de fossiles. Sur scène, au centre de la terre, on découvre un peuple de troglodytes "quelques têtes d'êtres bizarres, au front très déprimé, au regard fauve, aux cheveux ébouriffés, paraissent entre les rochers du fond, écoutant et donnant des signes de la plus extrême surprise $»^{15}$. Une expédition découvre donc un peuple entier d'hommes 
des premiers âges, sans qu'il soit proposé d'explication rationnelle à sa présence. Il s'impose comme réalité, non par une accumulation de données scientifiques vérifiables et historiques mais par une visualisation de scènes héritées de découvertes scientifiques. L'affirmation qu'il existe un feu central au centre du globe s'impose uniquement par l'expérience des héros, et non par raisonnement. A l'acte I, $5^{\circ}$ tableau («Le feu central»), les didascalies nous instruisent sur ce que voient les héros et donc les spectateurs : «La scène représente le centre de la terre. Partout des flammes, des gerbes étincelantes, laves incandescentes, coulent de toutes parts. Torrents de métaux liquides, argent et or, en fusion (...) Les phénix, les follets et les salamandres ont paru (...) Ils [les personnages] disparaissent au milieu des danses $»^{16}$. Les commentaires des critiques de l'époque insistent particulièrement sur la féerie que représente ce tableau. On relira avec intérêt cet extrait de l'étude de Robert Pourvoyeur :

dans la région du feu, s'exécute le ballet des salamandres, que Mortier n'aime pas beaucoup, mais que les autres critiques ont apprécié. Choufleri remarque les charmants « costumes ultra-collants, mi partie bleu marine et vieil or, avec les longs gants noirs et le casque à trois ailes noires. (...) très jolis aussi les papillons blancs, les chauves-souris en argent, et les quatre salamandres rouges ». Quant à l'étoile, elle a un corset de satin or et une jupe de gaze safran lamée d'or (Scapin). En fait d'accessoires nouveaux, il y a « des petites fontaines argentées » rappelant « celles de la place de la Concorde ». Mortier se plaint de ce « fouillis », « d'un tas de choses brillantes, scintillantes, miroitantes, éclatantes, d'une quantité d'accessoires de prix, le tout éclairé par une lumière crue à laquelle se mêlent, vers la fin, les lueurs rouges des flammes de Bengale (...) Stoullig est (...) très satisfait : " mise en scène curieuse aux tons cuivrés et argentés, qui évoquent un peuple de salamandres et de divinités souterraines $»^{17}$.

Le feu central est personnifié par des acteurs en tenues brillantes, jaunes et rouges, et un ballet symbolise le jeu des flammes et leurs ondulations. Pas de commentaires, pas de dialogues, juste du visuel et du spectaculaire. La science est une donnée immédiate et onirisée.

Les voyages successifs des personnages permettent d'illustrer l'extraordinaire réalité de théories scientifiques et de découvertes techniques. Sur scène, le rythme du spectacle demande un enchaînement des dialogues et des actions qui supporterait mal les monologues ou les expositions magistrales des savants verniens. L'auteur coupe tout rattachement des aventures que vivent ses personnages avec des données précises, vérifiables.

Il n'utilise pas le thème scientifique dans un but pédagogique, il veut distraire le public. Pour ce, Jules Verne va développer le côté ludique de la science : il met en scène à partir du savoir de son temps des expéditions sur une autre planète, au centre de la terre, sous les eaux.

Au théâtre, la science n'est pas l'objet de démonstrations ou de discussions, elle est visuelle et immédiate. Les décors illustrent des réalités scientifiques démontrées (feu central, sous-marin) ou supposées (existence de vie dans l'espace, histoire de l'Atlantide). Les costumes des danseurs et la mise en scène présentent les découvertes de la science en action, par des symbolisations concrètes (mouvement des flammes du centre de la terre, diversité des poissons sous les mers, dénuement des premiers hommes).

Jules Verne distrait son public par l'intégration dans un spectacle comique et tragique, musicale et parlé, dansé et statique d'une donnée scientifique onirisée. La science se dévoile sur l'instant et ses débordements imprègnent immédiatement l'imaginaire. 
G. Méliès, Les sirènes, Deux cent mille lieues sous les mers, photo de plateau

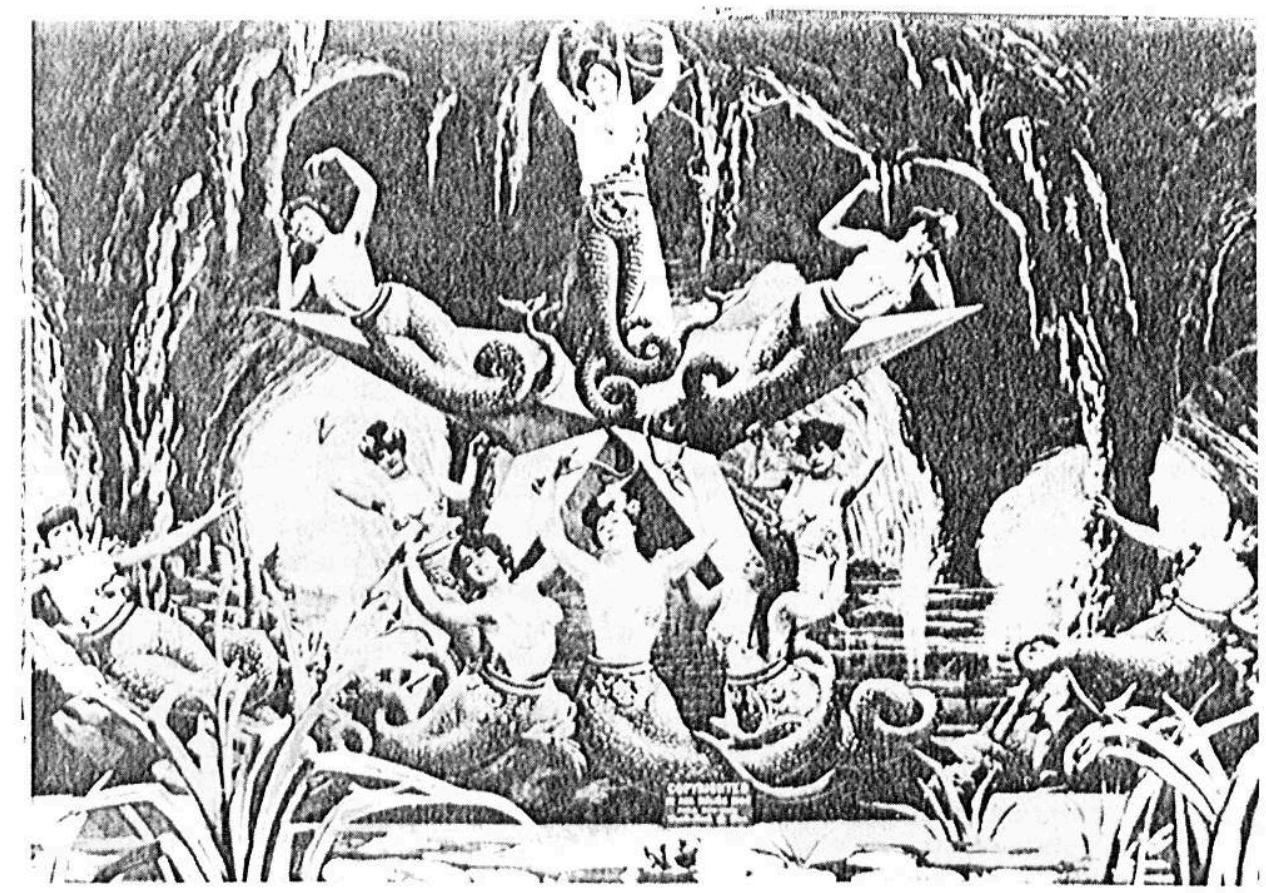

Coll. Malthète-Méliès

\section{NOTES}

1. François Raymond, « Avant-propos », in Voyage à travers l'impossible, Ed. J.J. Pauvert, Paris, 1981, p. 11.

2. Rappelons à ce sujet le texte de 1863, publié récemment, dans lequel Jules Verne décrit, sous le masque de la fiction, les grandes voies du théâtre contemporain. Le héros, Michel, est engagé dans le Grand Entrepôt Dramatique :

"C'était une merveilleuse organisation que celle du Grand Entrepôt Dramatique, il comprenait cinq grandes divisions : $1^{\circ}$ Haute comédie et comédie de genre. $2^{\circ}$ vaudeville proprement dit. $3^{\circ}$ drame historique et drame moderne. $4^{\circ}$ opéra et opéra-comique. $5^{\circ}$ revues, féeries (...) En trentesix heures, on pouvait livrer une comédie de genre ou une revue de fin d'année. (...) Il s'y trouvait des employés de talents, préposés l'un aux Expositions, l'autre aux Dénouements, celui-ci aux Sorties, celui-là aux Entrées de personnages; l'un tenait le bureau des rimes riches, quand on voulait absolument des vers, l'autre la partie des rimes courantes pour simple dialogue d'action. Il existait aussi une spécialité de fonctionnaires (...) [qui] avait pour mission de refaire les pièces des siècles précédents " (Paris au XXº siècle, Hachette, 1994, p. 172).

Par-delà l'évidente plaisanterie, ce roman de 1863 (année des débuts des romans verniens), stigmatise un mode de production théâtrale fait d'emprunts, de canevas éculés, de poncifs et de scènes inévitables. Jules Verne n'affirme rien d'autre qu'une esthétique particulière du théâtre qui associe des éléments pris çà et là, tantôt dans le drame moderne, tantôt dans le vaudeville, 
tantôt encore dans la comédie de genre. Le théâtre vernien est au croisement des formes artistiques mais aussi des genres.

3. Les Aventures d'Hatteras, Note de l'éditeur.

4. Voyage au centre de la terre, Folio Junior, Gallimard, Paris, 1980, p. 49.

5. Ibidem, p. 316

6. Voyage à travers l'impossible, op. cit., p. 65-66

7. Ibid, p. 65.

8. Rappelons les commentaires de Raymond Pourvoyeur sur la question :

«La partie comique - Tartelet et Valdemar - était représentée par les désopilants « Alexandre, pour qui j'ai depuis longtemps une vieille affection, et par le joyeux Dailly: ces deux artistes jouant pour la première fois ensemble, sont arrivés à dérider la salle, qui n'entrouvrait les dents qu'à leur apparition ». Mortier est du même avis que Noël (...) « Mon Dieu, si jamais je cours un grand danger, si je suis en mer sur le point de faire naufrage, en voiture emporté par un cheval affolé, en ballon au moment où le gaz s'en échappe par une déchirure fatale, fais - c'est un vœu bien égoïste que je forme là - fais que Dailly soit près de moi. (...) Avec Dailly en face de soi, on peut tout supporter. » Stoullig pour sa part, surenchérit : « Il n'y a pas à dire, Dailly a l'oreille du public : il paraît, le rire s'épanouit sur tous les visages comme il illumine sa large face (...) du haut en bas des galeries, chacun, indulgent et rasséréné, sent que le rire est le propre de l'homme. » » Op. cit. p. 90 .

9. Ibid. p. 19

10. Rappelons que la définition que donne Rousseau du mélodrame: « un genre de drame dans lequel les paroles et la musique, au lieu de marcher ensemble, se font entendre successivement, et où la phrase parlée est en quelque sorte annoncée par la phrase musicale ». Cité dans W.G. Hartog, Guilbert de Pixérécourt, Thèse, Paris, Champion, 1912, p. 40. Repris dans Le Drame, Michel Lioure, Coll. U, Armand Colin, 1963, p. 35.

11. Cité par Robert Pourvoyeur, "De l'extraordinaire à l'impossible», post-face de Voyage à travers l'impossible, op. cit. p. 94.

12. On se rappellera pour mémoire la description que fait Axel pour rendre la beauté du monde souterrain : "Le mot « caverne » ne rend évidemment pas la pensée pour peindre cet immense milieu. Mais les mots de la langue humaine ne peuvent suffire à qui se hasarde dans les abîmes du globe. (...) Toutes ces merveilles, je les contemplais en silence. Les paroles me manquaient pour rendre mes sensations. Je croyais assister, dans quelque planète lointaine, Uranus ou Neptune, à des phénomènes dont la nature «terrestrielle» n'avait pas conscience (...). J'admirai avec une stupéfaction mêlée d'une certaine quantité d'effroi » (Voyage au centre de la Terre, Gallimard, foliojeunesse, p. 206). Dans un roman, la beauté se décrit, au théâtre, elle se montre...

13. Voyage à travers l'impossible, Op. cit., p. 98

14. Le dix-neuvième siècle tente une compréhension des forces qui régissent l'univers, et c'est dans cette optique que se comprennent les études entreprises par les savants de l'époque. Pierre Simon Laplace propose une mécanique de l'espace qui rend compte de la formation et du fonctionnement du système solaire, Le Verrier et Adams démontrent mathématiquement, après l'étude de l'orbite curieuse d'Uranus, qu'un corps attire la planète à lui, et qu'il existe donc une huitième planète dans le système solaire (1846). On se passionne dès lors pour ces sphères, célestes et inaccessibles mais que la logique scientifique peut explorer. Fontenelle croyait aux extraterrestres, Arago à l'habitabilité du Soleil... Les tentatives pour connaître ou pour communiquer avec créatures de l'espace se multiplient. Gauss propose de tracer des symboles géométriques dans les plaines de Russie pour attirer l'attention des Sélénites, Cros en 1869, et plus tard Schmoll tentent d'envoyer des signaux lumineux vers les habitants de la Lune.

15. Voyage à travers l'impossible, Op. Cit., p. 28

16. Ibidem, p. 23

17. Robert Pourvoyeur, Op. Cit., p. 92 